\title{
Effect of Chondroitinase ABC on Purulent Sputum from Cystic Fibrosis and Other Patients
}

\author{
ISMAT A. KHATRI, K. RAMAKRISHNAN BHASKAR, J. THOMAS LAMONT, \\ S. UMADEVI SAJJAN, CATHERINE K.Y. HO, AND JANET FORSTNER
}

\begin{abstract}
Research Institute, Department of Structural Biology and Biochemistry, The Hospital for Sick Children, Toronto, Ontario, Canada [I.A.K., S.U.S., C.K.Y.H., J.F.F.]; Beth Israel Deaconess Medical Center, Boston, Massachusetts, U.S.A. [K.R.B., J.T.L.]
\end{abstract}

\begin{abstract}
Cystic fibrosis (CF) patients develop chronic lung infections associated with airway obstruction by viscous and insoluble mucus secretions. Although mucus glycoproteins (mucins) are thought to be responsible for mucus plugs, other glycoconjugate components of airway secretions have not been systematically evaluated. The aim of the present study was to determine whether chondroitin sulfate proteoglycans (CSPG) contribute to the insolubility of CF sputum. Sputa obtained from $18 \mathrm{CF}$ patients were incubated with chondroitinase $\mathrm{ABC}(\mathrm{ChABC})$ or buffer (control) for $18 \mathrm{~h}$ at $37^{\circ} \mathrm{C}$, and after centrifugation at $12,000 \mathrm{~g}$, the volume of the insoluble pellet and turbidity of the supernatant were determined as measures of solubility. ChABC caused a $70-90 \%$ reduction in supernatant turbidity and a $60-70 \%$ decrease in pellet volume of the 13 purulent CF sputa, but had much less effect on the five nonpurulent CF sputa tested. Similar results were obtained with two non-CF purulent and two non-CF, nonpurulent sputa. Gel electrophoresis, Western blot, and slot blot immunoassays with antichondroitin sulfate and antimucin antibodies revealed that purulent sputa ( $\mathrm{CF}$ and non-CF) contained more CSPG and less mucin than nonpurulent sputa. In vitro mixing experiments showed that mucin in nonpurulent
\end{abstract}

\section{ABSTRACT}

sputa was reduced upon incubation with purulent sputa, presumably because of degradation or a loss of immunoreactive mucin epitopes from leukocyte and/or bacterial enzymes present in purulent sputa. Our results suggest that CSPG contribute more significantly than mucins to the insolubility of purulent tracheobronchial secretions from CF patients. Because purulent sputa from non-CF patients showed a similar pattern, our observations with CF sputa may have wider applicability. (Pediatr Res 53: 619-627, 2003)
Abbreviations
CSPG, chondroitin sulfate proteoglycans
PG, proteoglycans
CS, chondroitin sulfate
DC, decorin
ChABC, chondroitinase $\mathrm{ABC}$
CF, cystic fibrosis
HLM, human lung mucin
TBM, human tracheobronchial mucin
GAG, glycosaminoglycans

$\mathrm{CF}$ is a life-threatening inherited disease caused by genetic mutation of the $\mathrm{CF}$ transmembrane conductance regulator (CFTR), a cyclic AMP-dependent chloride channel present in secretory and other cells throughout the body. Despite rapid advances in our knowledge of the structure and function of CFTR, the link between the mutations in this gene and the clinical manifestation of widespread obstruction of exocrine glands with viscous mucus secretions remains obscure.

Received September 19, 2001; accepted July 6, 2002.

Correspondence: Janet F. Forstner, M.D., Ph.D., The Hospital for Sick Children, 555 University Avenue, Toronto, ON M5G 1X8, Canada; e-mail: jfforst@sickkids.on.ca

Support for this study was provided by the Canadian Cystic Fibrosis Foundation and Select Therapeutics, Toronto, ON, Canada. C.K.Y.H. was the recipient of a summer student research award from Select Therapeutics, Toronto (1998) and the Canadian CF Foundation (1999)

DOI: 10.1203/01.PDR.0000054780.11755.B9
Almost all CF patients develop chronic lung disease related to airway obstruction, inflammation, and recurrent infections that are eventually lethal. In CF airways and intestine, goblet cell hyperplasia and mucin hypersecretion are characteristic, as is the presence of dilated airway submucous glands and intestinal crypt lumina (1-4). Inflammatory cell products stimulate airway secretions and thus contribute to mucus obstruction (4). Secretory mucin macromolecules, consisting of disulfidedependent polymers secreted by epithelial cells, are thought to be largely responsible for the viscoelastic gels and obstructive plugging of bronchial secretions. Another component that contributes to increased viscosity is DNA released from lysed neutrophils in the airways $(5,6)$. This has led to the clinical use of recombinant DNase to reduce sputum viscosity and relieve pulmonary obstruction in CF (7). Clinical improvement after DNase is often only temporary, and obstructive symptoms tend to recur. 
The potential role of PG in mucus plugging has received much less attention, even though there have been several reports that PG are secreted by airway epithelial cells $(8-17)$. With special reference to $\mathrm{CF}$, there is an increase in sulfated high molecular weight glycoconjugates secreted by CF airways in situ, in organ culture, and/or in cultured CF nasal epithelial cells $(10,11,15,18,19)$. Immortalized intrahepatic biliary epithelial (IBE) cell lines from CF patients also secrete significantly more CS than the same cells from non-CF patients (20). These in vitro findings raise the possibility that excessive production of PG may be directly linked to CFTR dysfunction. In addition, infection alone seems to stimulate secretion of CSPG, because LeTreut et al. (12) found elevated CSPG in the sputum of both $\mathrm{CF}$ and non-CF patients suffering from severe chronic bronchial hypersecretion. Rahmoune et al. (17) reported the presence of CSPG in sputum from infected CF sputa, but among the non-CF (chronic bronchitic) patients studied, two infected samples did not contain CSPG whereas a noninfected sample did. Thus, the causal relationships, if any, between infection, CFTR mutation, and PG secretion are still in need of clarification.

By virtue of their long linear oligosaccharides, negative charge, and adhesive properties (21), luminal PG might be expected to influence the rheologic properties of secretions. Under conditions of diminished hydration resulting from faulty chloride secretion for example, it can be speculated that hyperconcentration of PG (as well as mucins) would increase the tendency to develop tenacious and insoluble secretions. This consideration prompted us to determine the influence of CSPG on sputum solubility, to assess CSPG and mucin in CF sputum, and to determine whether purulent sputum affects the immunoreactivity of mucin. Results obtained with purulent CF sputa and a limited number of purulent sputa from non-CF patients were similar, suggesting that the role of CSPG in sputum insolubility may extend to purulent sputa associated with other diseases.

\section{MATERIALS AND METHODS}

Patient samples. Sputa from $18 \mathrm{CF}$ and four non-CF patients (aged 21-56 y) were collected with informed consent from patients at St. Michael's Hospital, Toronto, Canada (Table 1). As determined by the clinical microbiology laboratory, all sputa from CF patients (except one), were infected with Burkholderia cepacia and/or Pseudomonas aeruginosa, whereas all sputa from non-CF patients (except one with $P$. aeruginosa) were free of bacterial pathogens. Altogether there were 15 purulent (13 of which were CF) and seven nonpurulent (five of which were CF) samples. The term "purulent" was used only if samples appeared dark yellow or yellow-green, as sputum color has been shown to be a good indicator of the severity of bronchial disease, with gradations of color reflecting the activity of inflammatory agents (22). Eight additional sputum samples were collected with informed consent from patients in

Table 1. Patient data

\begin{tabular}{|c|c|c|c|c|c|c|}
\hline Purulent sputum & Diagnosis & Patient no. & Source (city) & Sex & Age & Lung function $\mathrm{FEV}_{1}(\%)$ \\
\hline No & $\mathrm{CF}$ & 1 & Toronto & M & 23 & 45 \\
\hline No & $\mathrm{CF}$ & 2 & Toronto & M & 21 & 73 \\
\hline No & $\mathrm{CF}$ & 3 & Toronto & M & 25 & 61 \\
\hline No & $\mathrm{CF}$ & 22 & Toronto & M & 34 & 93 \\
\hline No & $\mathrm{CF}$ & 23 & Toronto & $\mathrm{F}$ & 25 & 44 \\
\hline No & Asthma & 25 & Toronto & M & 42 & ND \\
\hline No & Acute quadriplegia & $\mathrm{SC} 3$ & Boston & $\mathrm{U}$ & $>20 *$ & ND \\
\hline No & Pneumonia (viral) & CL1 & Boston & $\mathrm{U}$ & $>20 *$ & ND \\
\hline No & Tracheobronchitis & CL2 & Boston & $\mathrm{U}$ & $>20 *$ & ND \\
\hline No & Asthma & CL3 & Boston & $\mathrm{U}$ & $>20 *$ & ND \\
\hline Yes & $\mathrm{CF}$ & 5 & Toronto & M & 32 & 24 \\
\hline Yes & $\mathrm{CF}$ & 11 & Toronto & M & 45 & 29 \\
\hline Yes & $\mathrm{CF}$ & 17 & Toronto & M & 33 & 21 \\
\hline Yes & $\mathrm{CF}$ & 18 & Toronto & M & 24 & 32 \\
\hline Yes & $\mathrm{CF}$ & 19 & Toronto & M & 28 & 33 \\
\hline Yes & $\mathrm{CF}$ & 20 & Toronto & $\mathrm{F}$ & 22 & 32 \\
\hline Yes & $\mathrm{CF}$ & 21 & Toronto & $\mathrm{F}$ & 38 & 35 \\
\hline Yes & $\mathrm{CF}$ & 4 & Toronto & M & 19 & 16 \\
\hline Yes & $\mathrm{CF}$ & 6 & Toronto & M & 23 & 33 \\
\hline Yes & Myasthenia gravis & 8 & Toronto & $\mathrm{F}$ & 41 & ND \\
\hline Yes & COPD & 10 & Toronto & $\mathrm{F}$ & 56 & 62 \\
\hline Yes & $\mathrm{CF}$ & CF1 & Boston & $\mathrm{U}$ & 35 & ND \\
\hline Yes & $\mathrm{CF}$ & CF2 & Boston & $\mathrm{U}$ & 23 & ND \\
\hline
\end{tabular}

$\mathrm{ND}$, not determined; $\mathrm{U}$, unknown; $\mathrm{COPD}$, chronic obstructive pulmonary disease; $\mathrm{FEV}_{1}$, forced expiratory lung volume per minute (\% predicted).

* Age between 20 and 40 years but otherwise unknown. 
Boston, MA, U.S.A. These included purulent sputa from two adult CF patients (CF1, CF2) at the Boston Childrens' Hospital, three nonpurulent sputa (SC1-SC3) from acute quadriplegic patients at the West Roxbury VA Medical Center, and three other non-CF nonpurulent samples (CL1-CL3) from intubated patients at the Brigham and Women's Hospital in Boston. All samples were frozen at $-20^{\circ} \mathrm{C}$ pending further processing.

Enzymes and antibodies for PG assays. Chondroitinase ABC lyase (protease-free, E.C. 4.2.2.4, Proteus vulgaris) and trypsin (bovine) were obtained from Sigma Chemical, St. Louis, MO, U.S.A. Rabbit polyclonal anti-human CSPG antibody (anti-CSPG) (MAb 2030) and mouse anti-human CSPG MAb (anti-CS) (MAb 2029) were obtained from Chemicon International, Temecula, CA, U.S.A. Anti-CSPG has been shown to recognize the unsaturated disaccharide glucuronic acid $N$-acetyl galactosamine sulfate attached to the core protein through the linkage tetrasaccharide Xyl-Gal-Gal-GlcU (23). This antibody was discontinued by the supplier partway through this study, thus anti-CS was substituted for subsequent experiments. The anti-CS antibody recognizes mature CSPG core glycoprotein of $250 \mathrm{kD}$ as well as precursor polypeptides of 210,220 , and $240 \mathrm{kD}$. A polyclonal rabbit antibody to the human CS-containing PG, DC (PG II) (anti-DC), was purchased from Biogenesis, Kingston, NH, U.S.A. This antibody was made against a synthetic peptide of human DC and recognizes human DC as well as native bovine and chicken DC. The antibodies were used in Western blot and/or slot blot immunoassays of sputum samples as described below.

Incubation of sputum with ChABC and trypsin. Frozen sputa were thawed on ice, the aqueous phase discarded and the gel phase evenly dispersed by shearing in $25 \mathrm{mM}$ Tris-acetate buffer $\mathrm{pH}$ 7.4. The dispersion was brought to a total volume of $2 \mathrm{~mL}$. Duplicate aliquots (approximately $500 \mu \mathrm{L}$ ) of each sputum sample were incubated for $18 \mathrm{~h}$ in buffer at $37^{\circ} \mathrm{C}$ with or without the addition of $1 \mathrm{U}$ ChABC or $6 \mathrm{U}$ of trypsin in a total volume of $1.2 \mathrm{~mL}$. After centrifugation at 12,000 rpm for 20 min at $4{ }^{\circ} \mathrm{C}$, the supernatant turbidity was determined by measuring absorbance at $600 \mathrm{~nm}$. A photograph of each insoluble pellet was scanned and digitized, and the area (as a measure of pellet volume) was quantified using the "Measurement Macros" function of National Institutes of Health version 1.60 .

Mucins and anti-mucin antibodies. Two different preparations of purified respiratory mucin and their respective antibodies were used. HLM, purified from noninfected, nonpurulent tracheal secretions of a patient with acute quadriplegia, and a MAb against HLM have been described earlier (24). A second human TBM was purified by the $\mathrm{CsCl}$ ultracentrifugation method of Carlstedt et al. (25) starting with the gel phase of pooled, purulent CF sputa (from 12 to 15 patients) homogenized in $6 \mathrm{M}$ guanidine $\mathrm{HCl}$. This method effectively separates mucins from $\mathrm{PG}$, lipids, DNA, and smaller proteins. Purity of TBM was verified by SDS-PAGE and amino acid and amino sugar analyses by the Picotag HPLC system (Waters Associates, Mississauga, ON, Canada). Carbohydrate profiles were obtained as described earlier (26) by anion exchange chromatography using a BioLC system (Dionex Corp., Sunnyvale, CA, U.S.A.) with pulsed amperometric detection. Poly- clonal anti-TBM was prepared in New Zealand rabbits, and immunoreactivity of the purified TBM confirmed by SDSPAGE and Western blotting using anti-TBM diluted 1:10,000 ( $\mathrm{vol} / \mathrm{vol})$. Immunolocalization was carried out on microscopic sections available from non-CF bronchial tissue (autopsy samples) as well as CF bronchial tissue (explants obtained at the time of double lung transplantation). For detection of mucin, anti-rabbit IgG-peroxidase conjugate was used as specified by the manufacturer (Vector Laboratories, Burlington, ON, Canada).

Sputum immunoassays. Equal volumes of supernatants or pellets from control (buffer alone) or ChABC-digested sputum were freeze dried, resuspended in SDS-PAGE reducing sample buffer, boiled for $3 \mathrm{~min}$, and subjected to SDS-PAGE and Western blotting. Gels were run in duplicate, one of which was blotted with anti-CSPG and the other with anti-HLM antibodies. Detection was carried out using a second antibody-alkaline phosphatase system (Roche Diagnostics, Laval, QC, Canada). The anti-HLM antibody has previously been used to identify CSPG in biliary epithelial cell secretions (20), which were therefore used in the present study as positive controls.

Slot blot immunoassays were performed on ChABCdigested sputum supernatants $(25$ and $50 \mu \mathrm{L})$ using anti-DC $(0.1 \mathrm{mg} / \mathrm{mL})$ and anti-CS (1:200 vol/vol) antibodies to measure CSPG. Mucin was assayed (in $50 \mu \mathrm{L}$ of $1: 10$ and 1:20 dilutions) using anti-TBM antiserum (dilution 1:10,000 vol/vol). Day-to-day variations in assay results were minimized by assaying one selected sputum sample (as a standard) on each day an experiment was performed. Values that fell on the linear range of immunoreactivity curves were used to calculate density. Results were expressed as slot density per unit supernatant volume or per microgram protein, the latter measured using the bicinchoninic assay (27).

Cellulose acetate electrophoresis of sputum GAG. Supernatants from four representative sputa (patients $4,11,8$, and 14; Table 1) were subjected to cellulose acetate electrophoresis according to published methods (28). Electrophoresis was carried out in an LKB 2117 multifor apparatus in $1 \mathrm{M}$ barium acetate buffer $\mathrm{pH} 5$. Titan III cellulose acetate plates $(60 \times 76$ $\mathrm{mm}$ ) were purchased from Helena Laboratories (Beaumont, TX, U.S.A.). Plates were stained in a solution of alcian blue ( $1 \%$ in water) for $10 \mathrm{~min}$ and destained with several changes of $5 \%$ acetic acid. GAG were identified by comparison with purified CS A, B, and C standards from Sigma Chemical.

Assays of mucin after incubation of nonpurulent with purulent sputum (mixing experiment). Equal volumes (100 $\mu \mathrm{L}$ ) of the supernatants from nonpurulent (patients 2 or 14 , Table 1) or purulent (patients 4 or 12, Table 1) sputa were incubated separately or together. The total volume in each incubation was made up to $250 \mu \mathrm{L}$ with buffer $(25 \mathrm{mM}$ Tris acetate $\mathrm{pH} 7.4$ ). After $3 \mathrm{~h}$ at $37^{\circ} \mathrm{C}$, the incubation mixtures were boiled for $3 \mathrm{~min}$, diluted with an equal volume of reducing sample buffer, and three aliquots $(50 \mu \mathrm{L}, 100 \mu \mathrm{L}$, and $200 \mu \mathrm{L})$ subjected to slot blot mucin immunoassays using anti-TBM $(1: 10,000 \mathrm{vol} / \mathrm{vol})$. Blots were digitally scanned and densitometric values were recorded. Mucin values that fell on the linear portion of the mucin standard curve were used to calculate density per unit volume of incubation mixtures. 
Statistics. Statistical analyses were performed by the twotailed $t$ test of paired or unpaired samples (StatView Student v. 1.0, SAS Institute, Cary, NC, U.S.A.). The level for statistical significance was $p<0.05$.

\section{RESULTS}

Standardization and reliability of sputum preparations for assay. Because of heterogeneous physical properties and variable composition, sputum has to be evenly dispersed to allow uniform sampling of multiple aliquots. To show that consistent and reproducible sampling was feasible, the gel phase of 22 different sputum samples was evenly dispersed by shearing up and down in a wide-bore pipette and then incubated for $15 \mathrm{~min}$ at $37^{\circ} \mathrm{C}$ in a rotary shaker with an equal volume $(0.5-1.0 \mathrm{~mL})$ of $0.2 \mathrm{M}$ Tris acetate buffer $\mathrm{pH}$ 7.4. Dispersed sputum was then centrifuged, and the supernatant turbidity (OD $600 \mathrm{~nm}$ ) and pellet volume of duplicate or triplicate samples were measured. Intrasample differences were $<10 \%$, as were differences between samples taken on different days from the same original sputum. These results (not presented) provided assurance that sampling was reproducible from sputum gel dispersions. As expected, purulent samples were always more gel-like and insoluble, and gave much higher (5- to 10-fold) supernatant turbidity and pellet volumes than nonpurulent samples.

Chondroitinase $A B C$ increases the solubility of $C F$ sputum. Dispersed sputum samples were incubated in duplicate with ChABC or with Tris acetate buffer (controls) for $18 \mathrm{~h}$ at $37^{\circ} \mathrm{C}$. As shown for a representative purulent sample (Fig. 1) ChABC caused visible clarification of the supernatant after centrifugation and a reduction in pellet volume. In the absence of enzyme, the supernatant remained semi-opaque and the
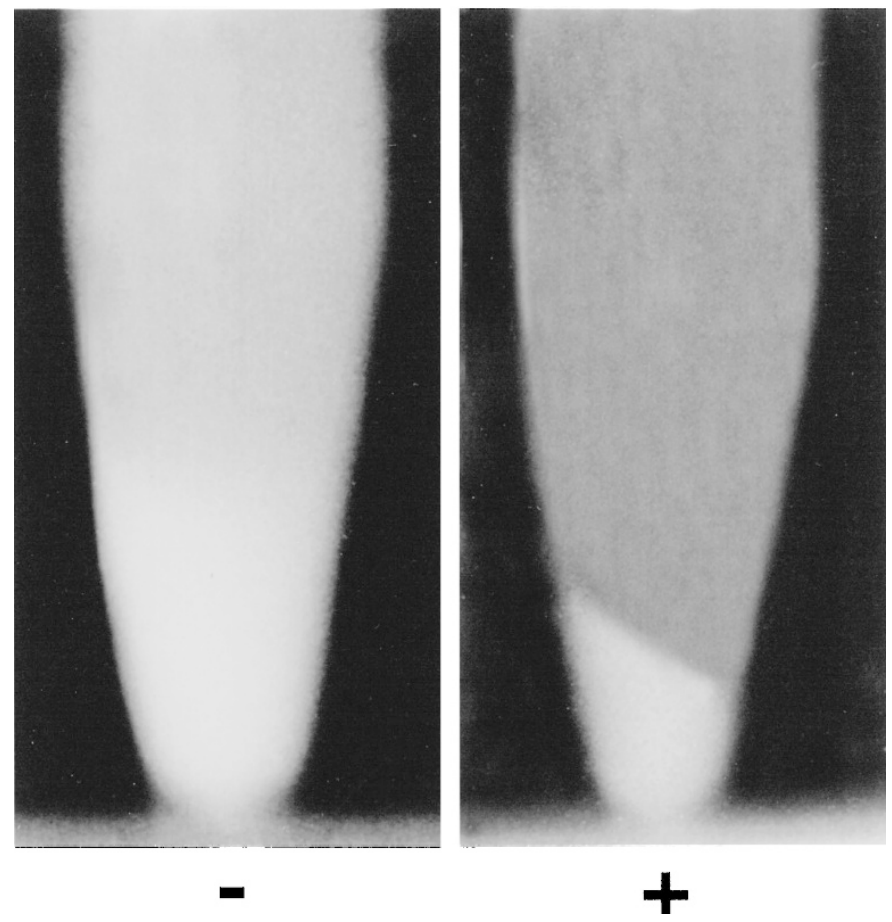

Figure 1. Clarification of sputum by $\mathrm{ChABC}$ digestion. The gel phase of purulent sputum was incubated for $18 \mathrm{~h}$ at $37^{\circ} \mathrm{C}$ in the presence $(+)$ or absence $(-)$ of $1 \mathrm{U}$ of $\mathrm{ChABC}$, and then centrifuged at $12,000 \mathrm{rpm}$ for $20 \mathrm{~min}$ at $4^{\circ} \mathrm{C}$. gel-like pellet volume was much larger. Quantification of this phenomenon was carried out on 18 CF sputa (13 purulent and five nonpurulent) and four non-CF sputa (two nonpurulent, two purulent) by measuring absorbance of supernatants at $600 \mathrm{~nm}$ (an index of light scattering by particulate material in solutions or suspensions) and pellet area (to reflect pellet volume). Purulent sputa showed significantly lower turbidity (Fig. 2a) and pellet volume (Fig. 2b) (85-90\% reduction in supernatant turbidity, $60-65 \%$ reduction in pellet volume) after enzyme digestion. The responses of two non-CF purulent samples included in this study could not be distinguished from those of the CF purulent sputa. Likewise, two non-CF, nonpurulent samples were indistinguishable from five $\mathrm{CF}$ nonpurulent sputa. Thus, the high turbidity of sputum samples and clarification by $\mathrm{ChABC}$ seemed to be associated with purulence rather than $\mathrm{CF}$ status.

Incubation of two purulent sputa (one $\mathrm{CF}$, one non-CF) with $6 \mathrm{U}$ of trypsin under the same conditions of time, temperature, and $\mathrm{pH}$ did not decrease solubility. Instead, trypsin caused a $30 \%$ increase in supernatant turbidity (not presented), presum-
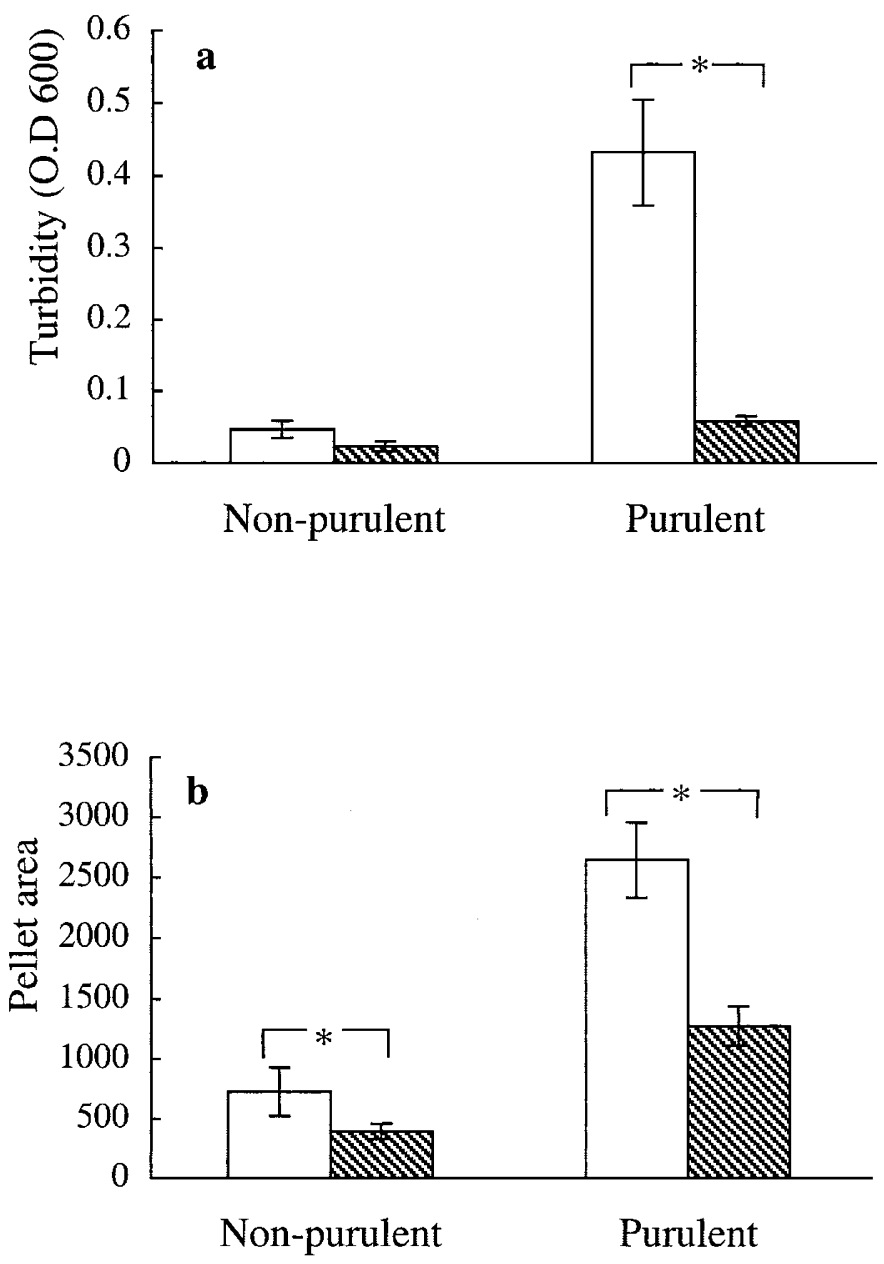

Figure 2. Effect of ChABC on sputum solubility. Equal aliquots of dispersed sputa (gel phase) of 15 purulent and seven nonpurulent samples (Toronto patients, Table 1) were incubated for $18 \mathrm{~h}$ at $37^{\circ} \mathrm{C}$ in buffer (open bars), or buffer containing ChABC (hatched bars). Samples were centrifuged and supernatant turbidity (OD 600) $(a)$ and pellet area (pixels) $(b)$ were measured. Values represent the mean \pm SEM for each group of samples. Asterisks denote statistically significant differences $[p<0.0001$ for $(a)$ and 0.001 for $(b)]$. 
ably as a result of the liberation of peptides from proteins, indicating that the solubilizing effect of $\mathrm{ChABC}$ was not mimicked by proteolytic degradation of sputum constituents.

Identification of $\boldsymbol{C S}$ in $\boldsymbol{C F}$ sputa. Western blot analyses of supernatants from chondroitinase-digested purulent $\mathrm{CF}$ sputa showed immunoreactive bands with anti-CSPG (Fig. 3a). The same bands were not detected in the supernatants of corresponding buffer-incubated controls. Immunoreactive bands of the same size range (118 kD, $90 \mathrm{kD}$, and below) were also seen in the corresponding pellets from each patient, but these were much less intense (results not shown), suggesting that after ChABC digestion, the bulk of CS was in the supernatant. Non-CF, nonpurulent samples gave very poor or negligible CS staining in supernatants (Fig. 3b) and pellets (not presented).

Serous cells from airway submucosal glands have been shown to synthesize DC, a PG also known as PGII or PG40, which contains CS B (dermatan sulfate) $(14,29,30)$. Therefore Western blot analyses of sputa were also carried out using anti-DC. The supernatants of ChABC-digested purulent CF sputa (patients 4 and12, Fig. $3 c$ ) showed an intense immunoreactive band at approximately $55 \mathrm{kD}$, the expected size of the core protein of intact DC $(30,31)$, and also less intense bands at lower molecular weights (possibly degraded products). Immunoreactivity was not seen in the case of nonpurulent (CF and non-CF) sputa (Fig. 3d). Slot blot immunoassays of CS were also performed on three nonpurulent samples (patients 14,22 , and 25) and 13 purulent samples (patients 4, 8, 10-13, 15-21). The mean immunoreactive DC content was 1.6 times higher in purulent than in nonpurulent samples $(2323 \pm 144$ versus $1460 \pm 298$ density units per $50 \mu \mathrm{L}$ ). Results of anti-CS slot blot immunoassays exhibited the same trends (1747 \pm 164 versus $1167 \pm 170$ units per $50 \mu \mathrm{L}$ ) in purulent versus nonpurulent samples. No CF-specific differences were discerned. These findings support the interpretation that purulence rather than CF status correlates best with an increase in CS in sputa.

Identification of mucin in CF sputa. In contrast to strong anti-CSPG reactivity, the majority of supernatants from ChABC-digested purulent $\mathrm{CF}$ sputa did not react, or reacted

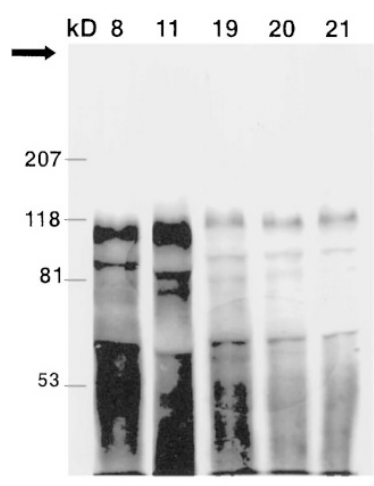

(a)

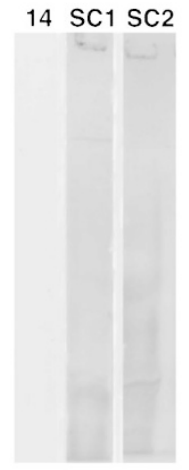

(b)

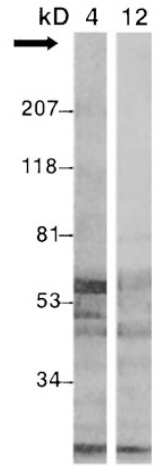

(c)

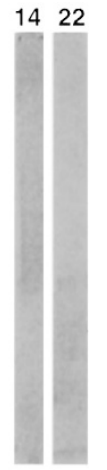

(d)
Figure 3. Western blotting of sputum samples using antibodies to PG. Supernatants from ChABC-digested purulent $(a, c)$ and nonpurulent $(b, d)$ sputa were subjected to SDS-PAGE followed by Western blotting using anti-CSPG antibody $(a, b)$ and anti-DC antibody $(c, d)$. Numbers (top) identify the patients listed in Table 1. Arrow marks the gel origin. only weakly, with the MAb against HLM (anti-HLM). These results are shown in Figure 4, $a$ and $b$. None of the purulent CF samples examined (patients 11, 19, 20,21) showed significant immunoreactivity with anti-HLM antibody. Interestingly, one purulent non-CF sample (patient 8) also failed to show reactivity, again implying that low mucin reactivity is associated with purulence of sputum rather than CF status. As shown for nonpurulent, non-CF sputa however (Fig. 4b) strong reactivities (dark smears) were seen with anti-HLM. More than $80 \%$ of the anti-HLM reactivity was in the supernatant, indicating that the mucin in these samples was mainly in a soluble form, rather than in the insoluble gel phase or pellet.

Characterization of purified CF TBM and its polyclonal antibody, anti-TBM. Because the anti-mucin antibody used above was raised to a non-CF mucin, we considered the possibility that apparent low reactivity of mucin in purulent $\mathrm{CF}$ sputa might be the result of intrinsic, CF-specific differences in mucin epitopes. TBM was therefore purified from pooled CF purulent sputa as described in "Methods." By SDS-PAGE and silver staining, the TBM preparation appeared as a high molecular mass band at the top of the gel $(>250 \mathrm{kD})$ and no other bands were detected (Fig. 5, lane $b$ ). The amino acid profiles of the two mucins HLM and TBM were almost identical (Table 2), but comparison of sugar profiles revealed that the CF mucin contained more fucose, GlcNAc and Gal, and less GalNAc and sialic acid than HLM.

A rabbit antiserum to purified TBM gave a positive signal in Western blots in the upper region of the gel (Fig. 5, lane c). Immunolocalization in both non-CF and CF bronchial sections revealed that the signal was present in goblet cells (not shown). There was no apparent difference in the signal intensity between the $\mathrm{CF}$ and non-CF tissue, although the number and size of the goblet cells were increased in the CF sections.

Dilutions of purulent and nonpurulent sputum (supernatants) were used to establish a standard curve for TBM (not presented) and to select appropriate dilutions of sample to ensure that values would fall on the linear range of the curve in subsequent mucin slot blot immunoassays.

Immunoreactive mucin in sputum samples. Sputum samples were first grouped into those with low (A) (0.01-0.07),

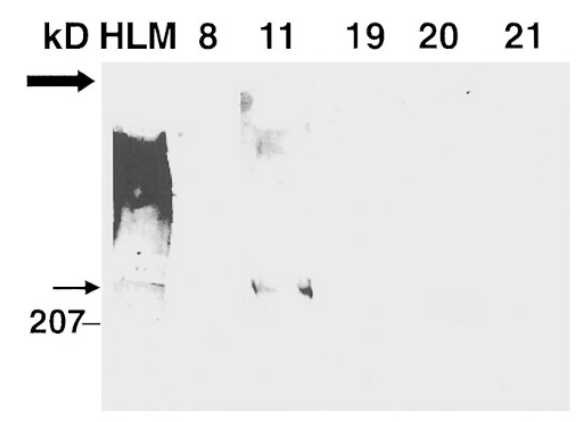

(a)

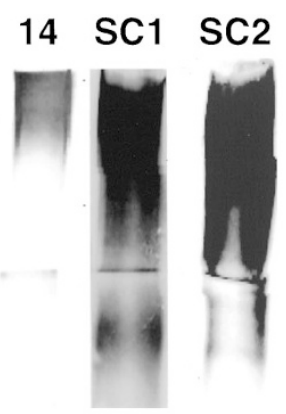

(b)
Figure 4. Western blotting of sputum samples using antibody to mucin. Supernatants from ChABC-digested purulent $(a)$ and nonpurulent $(b)$ sputa were subjected to SDS-PAGE followed by Western blotting using anti-HLM. Purified HLM was used as a positive control. Numbers (top) correspond to patients listed in Table 1. Thick arrow marks the origin and thin arrow marks the junction of stacking and separating gels. 


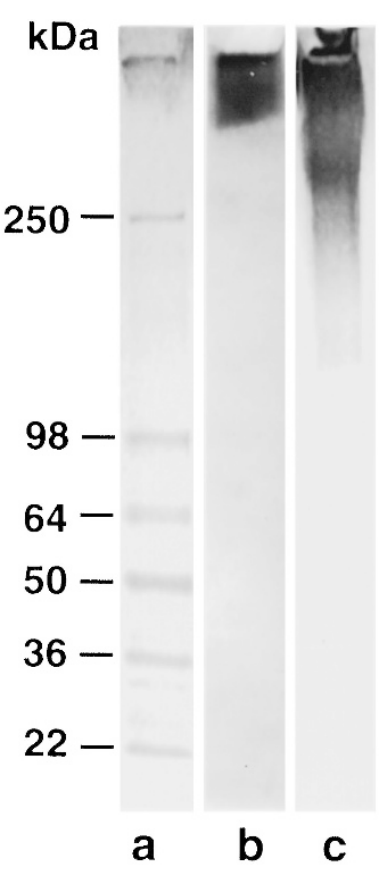

Figure 5. SDS-PAGE and Western blotting of purified TBM. Lane a represents prestained molecular mass standards, lane $b$ shows silver staining, and lane $c$ shows Western blots with anti-TBM antibody at a dilution of 1:10,000 ( $\mathrm{vol} / \mathrm{vol})$.

Table 2. Composition of respiratory mucins HLM and TBM (mol $\%)$

\begin{tabular}{lcc}
\hline & HLM & TBM \\
\hline Carbohydrates & 13.9 & \\
Fuc & 27.9 & 21.9 \\
Gal & 27.8 & 32.4 \\
GlcNAc & 12.7 & 35.1 \\
GalNAc & 17.7 & 7.7 \\
NAcNeu & & 2.9 \\
Amino acids & 6.6 & \\
Asp & 9.5 & 7.1 \\
Glu & 12.1 & 8.2 \\
Ser & 10.6 & 11.3 \\
Gly & 2.8 & 8.2 \\
His & 4.1 & 1.8 \\
Arg & 19.5 & 2.6 \\
Thr & 8.0 & 19.1 \\
Ala & 12.1 & 8.9 \\
Pro & 1.8 & 12.6 \\
Tyr & 4.8 & 1.0 \\
Val & ND & 5.2 \\
Met & 2.8 & 0.6 \\
Cys (CMC) & ND & 0.7 \\
Ile & ND & 2.8 \\
Leu & 2.4 & 5.5 \\
Phe & 2.9 & 1.9 \\
Lys & 36.8 & 1.8 \\
\hline
\end{tabular}

HLM values were calculated from Ref. 36. For HLM, the Ile and Leu peaks were obscured by amino sugar peaks and could not be calculated (ND). All values represent the average of two independent analyses.

intermediate (B) (0.12-0.26), and high (C) (0.34-1.04) turbidity as represented in Figure $6 a$. From each ChABC-digested supernatant $(1.2 \mathrm{~mL})$, two or three dilutions were assayed for total protein concentration and mucin (anti-TBM immunoreactivity). As shown in Figure $6 b$, the protein concentration

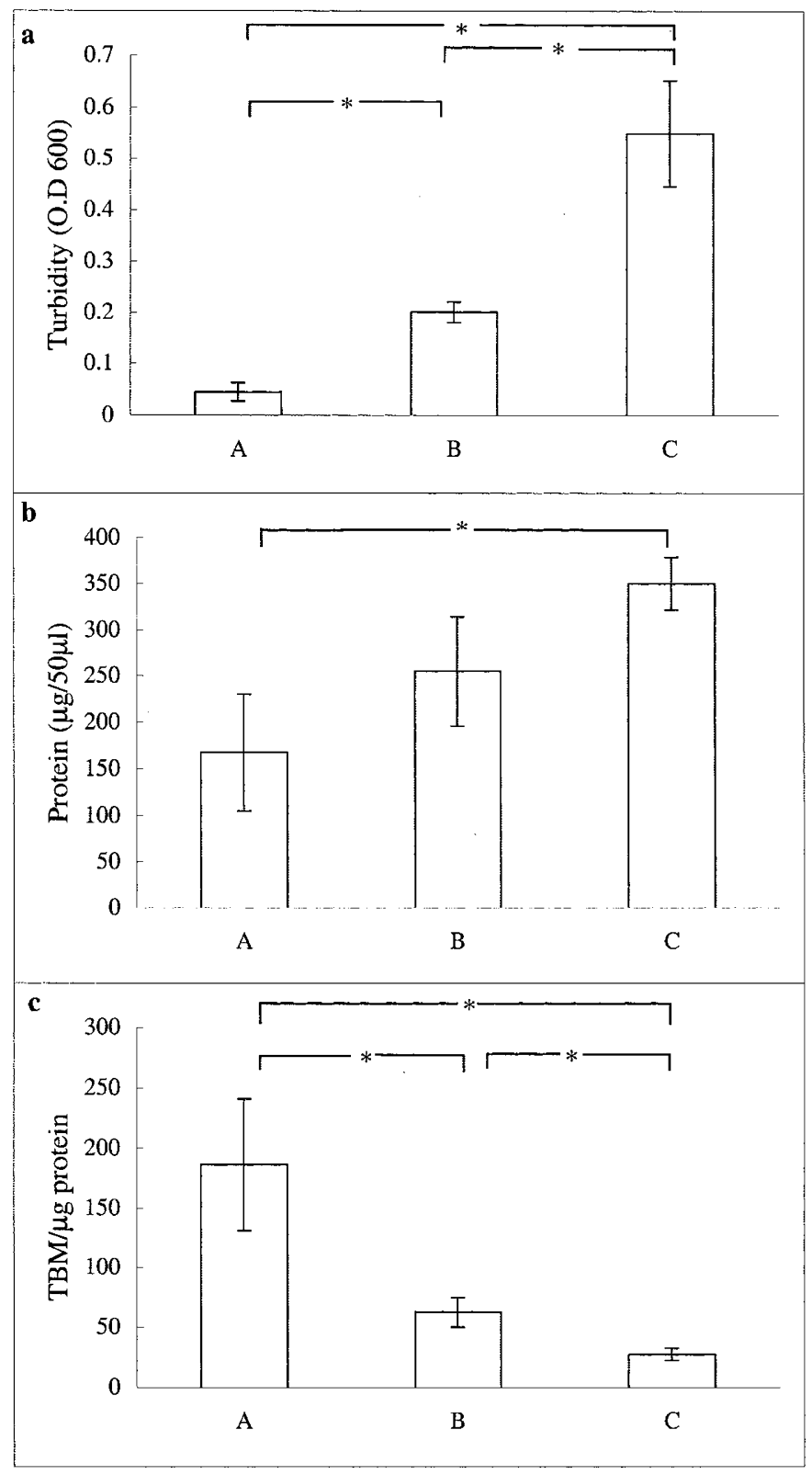

Figure 6. Turbidity, protein, and mucin (TBM) assays of sputum samples. Sputum supernatant samples in group A were from patients 1, 14, 22, and 25; in group $\mathrm{B}$ from patients $4,13,15,16,17,18$, and 20 ; and in group $\mathrm{C}$ from patients $8,10,11,12,19$, and 21 . The groups reflect low, intermediate, and high turbidity, respectively. Mucin was measured by slot blot immunoassay. Values represent mean \pm SEM (bars) and asterisks indicate $p<0.005$ for panel $a, p<0.02$ for panel $b$, and $p<0.03$ for panel $c$.

increased with increasing turbidity of samples. However, mucin immunoreactivity decreased as a function of unit protein (Fig. 6c), such that the most turbid (most purulent) group of sputa (C) had the lowest mucin.

The most likely explanation for the decreased mucin was that mucin antigenic epitopes were lost or the mucin was degraded by proteases and/or glycosidases of inflammatory cells or bacteria in purulent sputa (32-34). A test for the action of purulent sputa was carried out by incubating purulent (100 $\mu \mathrm{L})$ or nonpurulent $(100 \mu \mathrm{L})$ sputum supernatants separately (controls) or together. All incubations were adjusted to a final 
volume of $250 \mu \mathrm{L}$ with Tris acetate $\mathrm{pH} 7.4$ buffer and carried out for $3 \mathrm{~h}$ at $37^{\circ} \mathrm{C}$. Immunoreactivity of mucin was measured by slot blot immunoassay and the results expressed as mucin per $50 \mu \mathrm{L}$. If no loss had occurred, the immunoreactivity of mixtures should have equaled the sum of mucin densities in purulent and nonpurulent samples (i.e. the sum of black and white bars of Fig. 7). However, the measured mucin in mixtures (hatched bars) was lower in each case, indicating that mucin immunoreactivity in nonpurulent sputum was decreased after exposure to purulent sputum. Presumably, this also occurs in vivo after mucins are secreted into airways and encounter inflammatory cells. Because the identity of the major antigenic epitopes for anti-TBM are unknown at the present time, it is not clear whether the decrease in immunoreactivity reflects losses of oligosaccharide components or poorly glycosylated peptide regions of the mucin.

Identification of GAG in purulent sputa: Supernatants from four ChABC-digested purulent sputa were examined by cellulose acetate electrophoresis in an effort to identify the major type of CS chains (Fig. 8). Although the intact GAG used as standards differ structurally from the GAG fragments expected after $\mathrm{ChABC}$ digestion of sputa, alcian blue-positive spots were seen at approximately the same position as the commercial standard CS B (dermatan sulfate). Reactivity was strong in the three purulent samples (patients 4,11 , and 8), which is consistent with the earlier-demonstrated presence of DC in purulent samples. No alcian blue staining was noted in a sample of nonpurulent sputum (patient 14), which is consistent with earlier results showing low turbidity and poor reactivity with anti-CSPG and anti-DC in nonpurulent samples. The identity of the spot near the origin (in patients 4 and 8 ) remains to be established.

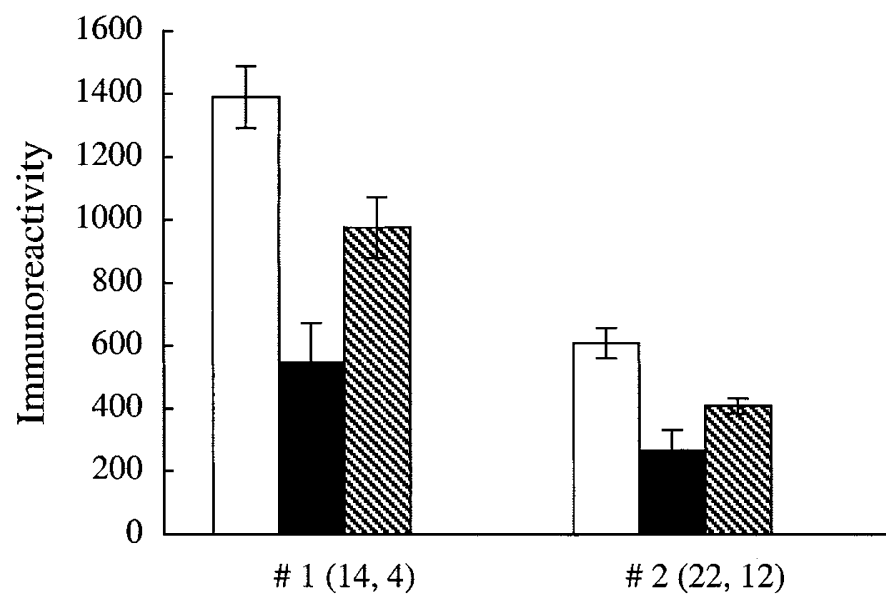

Figure 7. Loss of mucin immunoreactivity in purulent sputa. In two experiments (\#1, \#2) supernatant samples $(100 \mu \mathrm{L})$ of nonpurulent (white) and purulent (black) sputa were incubated separately or together (hatched) for $3 \mathrm{~h}$ at $37^{\circ} \mathrm{C}$ in Tris-acetate buffer $\mathrm{pH} 7.4$. The final volume of all incubations was $250 \mu \mathrm{L}$. Mucin was measured in duplicate samples of three dilutions by slot blot immunoassay using anti-TBM, and expressed as density per $50 \mu \mathrm{L}$. Bars show total range of values obtained. Numbers in brackets refer to patients listed in Table 1.

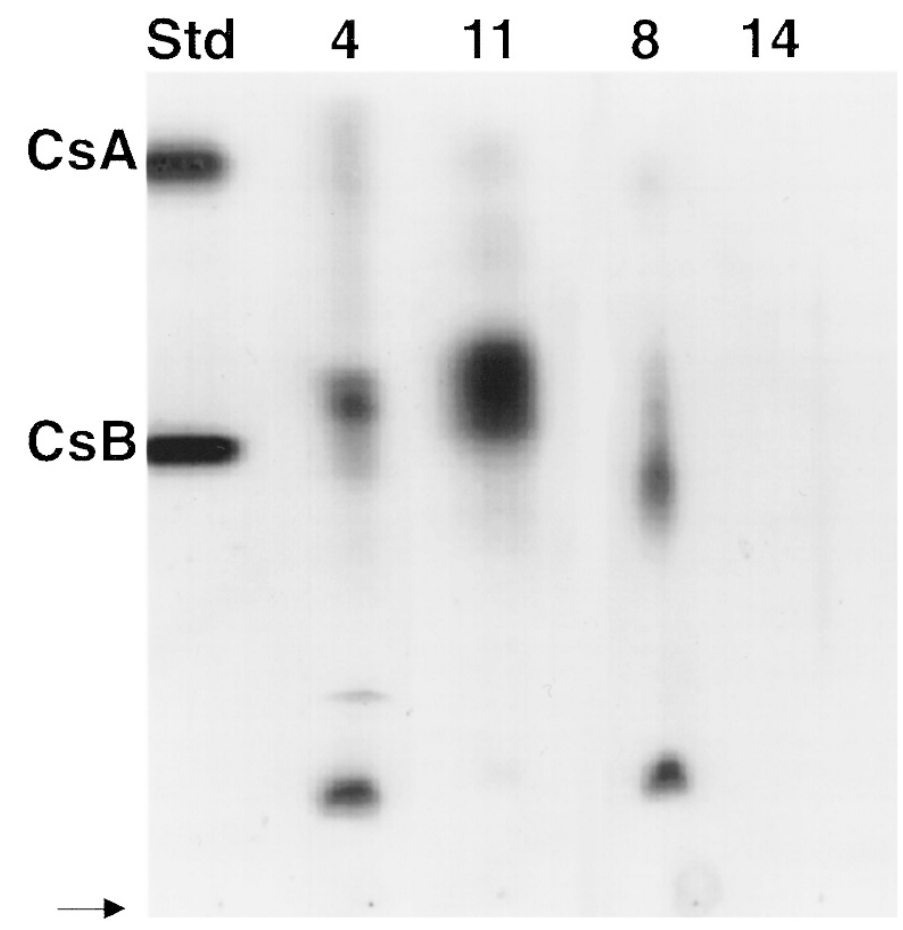

Figure 8. Cellulose acetate electrophoresis of purulent sputum. Supernatants from four representative sputa were subjected to discontinuous electrophoresis followed by alcian blue staining for the detection of GAG. Arrow (left) indicates point of sample application. CsA (chondroitin sulfate A) and CsB (dermatan sulfate) represent GAG standards. Numbers on top correspond to patients listed in Table 1.

\section{DISCUSSION}

Sputum turbidity and CSPG. The major finding of this study was that incubation with chondroitinase had a pronounced solubilizing effect on CF purulent sputa. The supernatant became almost transparent and the pellet size decreased dramatically. Although the non-CF patient population was small, there was no obvious trend to suggest that high turbidity and clearance by $\mathrm{ChABC}$ were unique to $\mathrm{CF}$ sputa. Non-CF purulent samples were equally affected by the enzyme.

CS in CSPG consist of long, unbranched polymeric chains of disaccharides (35). Digestion with the endoglycosidase ChABC leaves a modified disaccharide, delta unsaturated glucuronic acid- $N$-acetylgalactosamine, (GlcU-GalNAc), attached to the core protein through the linkage tetrasaccharide, glucuronic acid $\beta 1,3$ Gal $\beta 1,3$ Gal $\beta 1,4$ Xylose (35). The polyclonal anti-CSGP used in the initial Western blots in the present study recognizes the unsaturated disaccharide resulting from ChABC digestion (23). The anti-CS antibody recognizes mature CS PG core glycoprotein as well as the precursor polypeptides, and anti-DC antibody recognizes human DC. Western blots or slot blot immunoassays using the three different antibodies all showed that purulent sputa were enriched in CSPG content.

Solubilization was not improved with trypsin, indicating that higher protein (nonmucin, non-PG) content of purulent samples was unlikely to be responsible for their high turbidity. It is also unlikely that mucins in the sputa were responsible for turbidity inasmuch as ChABC does not degrade mucins (36) and mucins do not contain uronic acid. 
Possible mechanism of clarification of sputum by chondroitinase. CS chains range in length from 50 to 150 residues (molecular mass 20-50 kD) (21), and are therefore much longer and heavier than the individual branched O-linked oligosaccharides typical of mucins (1-20 residues) (37). Enzymatic digestion of CSPG is thus likely to disrupt complexes or large aggregates formed by the intertangling of long GAG chains with other macromolecules, including proteins, lipids, mucins, DNA, or other PG $(21,38,39)$. Presumably, the sputum solubilization observed in our studies was caused by dissociation of such aggregates.

The source of elevated CSPG in purulent sputa. The specific cell source(s) of PG in purulent sputum was not investigated, although the increase in DC content suggests that hypersecretion from serous cells of tracheal and bronchial submucosal glands may represent one source, inasmuch as CS-containing DC is a major product of these cells $(14,29$, 30). Other CSPG may also be produced by serous cells, however $(14,16,40)$, and other potential sources of CSPG include fibroblasts, inflammatory cells, chiefly leukocytes (4143), or leakage of extracellular matrix proteins (13) through damaged airway epithelium. The increase in CSPG content in purulent versus nonpurulent sputa suggests that bacterial infection stimulates PG synthesis and/or secretion. There are several published reports that are consistent with this suggestion. For example, Sommerhoff et al. (44) have reported that elastase and cathepsin $\mathrm{G}$ of inflammatory cells act as potent stimulants of CSPG release from serous cells. Purulent tracheobronchial secretions of patients with $\mathrm{CF}$ and other diseases are more highly sulfated and exhibit stronger acidic properties than those from health patients, which suggests an increase in CSPG $(10-12,15,45)$.

Low amounts of measurable mucin in purulent sputa. It is generally assumed that viscoelastic mucin macromolecules are responsible for the poor clearance of $\mathrm{CF}$ secretions from infected airways (46). In the present study, however, the measured mucin immunoreactivity was greater for nonpurulent than purulent sputa. It was considered highly unlikely that there would be an overall decrease in mucin synthesis and secretion in infected airways, because goblet cell number and secretion are almost always increased with infections. The decrease of mucin in purulent sputa also could not be attributed to intrinsic epitope differences between $\mathrm{CF}$ and non-CF mucins, because both anti-HLM and anti-TBM gave the same qualitative results in Western blots and slot blot immunoassays, despite recognizable mucin carbohydrate differences. The mixing experiment, in which mucin immunoreactivity in nonpurulent sputum decreased after a brief (3-h) incubation with purulent sputum, provides a reasonable explanation of the observed differences. Bacterial and/or inflammatory cell products present in purulent sputum, such as proteinases and/or glycosidases, are probably responsible for decreased mucin reactivity. This interpretation is supported by earlier reports that mucin degradation is accelerated during $P$. aeruginosa infections (34). Degradation could include removal of carbohydrate epitopes and/or "exposed" (poorly glycosylated) terminal regions of the mucin polypeptide (37). Because gel formation depends on end-to-end polymerization of mucin monomers, proteolytic excisions in terminal regions of monomers can cause mucin gels to collapse.

Clinical implications. The response of purulent sputa to ChABC suggests that mucins may not be the most important factor in causing the physical abnormalities of purulent tracheobronchial secretions. Agents such as $\mathrm{N}$-acetyl cysteine derivatives (47), gelsolin (48), dextran (49), and heparin are thought to have "mucolytic" properties, but evidence that these agents cause sustained relief from airway obstruction in chronic lung diseases is lacking. The present study suggests instead that PG GAG degradation may be warranted as a potential adjunct to therapy to improve airway clearance in chronic respiratory infections in general and in $\mathrm{CF}$ in particular.

Acknowledgments. The authors thank Dr. E.Tullis and Ms. A. Tsang, St. Michael's Hospital, Toronto, for sputum samples; Dr. M. Corey, the Hospital for Sick Children, Toronto, for patient clinical data and assistance with statistical analyses; and Dr. E. Cutz, the Hospital for Sick Children for bronchial sections.

\section{REFERENCES}

1. Snouwaert JN, Brigman KK, Latour AM, Malouf NN, Boucher RC, Smithies O, Koller BH 1992 An animal model for cystic fibrosis made by gene targeting. Science 257:1083-1088

2. Kent G, Oliver M, Kevin Foskett J, Frndova H, Durie P, Forstner J, Forstner GG, Riordan JR, Percy D, Buchwald M 1996 Phenotypic abnormalities in long-term surviving cystic fibrosis mice. Pediatr Res 40:233-241

3. Davis P, Drumm M, Konstan MW 1996 Cystic fibrosis. Am J Respir Crit Care Med 154:1229-1256

4. Puchelle E, Zahm J, Bentzmann S, Gaillard D 1997 Mucus and airway epithelium alterations in cystic fibrosis. In: Rogers DF, Lethem MI (eds) Airway Mucus: Basic Mechanisms and Clinical Perspectives. Birkhauser Verlag, Basel, Switzerland, pp 301-326

5. Bhaskar KR, Reid L 1980 Application of density gradient methods to study the composition of sol and gel phases of CF sputa and the isolation and characterization of epithelial glycoprotein from the two phases. In: Sturgess JM (ed) Perspectives in Cystic Fibrosis. Proceedings of the 8th International Cystic Fibrosis Congress, Toronto, Canada, pp 113-121

6. Potter JL, Spector S, Mathews, LW Lemm J 1969 Studies on Pulmonary secretions The nucleic acids in whole pulmonary secretions from patients with cystic fibrosis, bronchiectasis and laryngectomy. Am Rev Respir Dis 99:909-916

7. Stern M, Alton EW 1997 Therapeutic approaches to the lung problems in cystic fibrosis. In: Rogers DF, Lethem MI (eds) Airway Mucus: Basic Mechanisms and Clinical Perspectives. Birkhauser Verlag, Basel, Switzerland, pp 341-364

8. Bhaskar KR, O'Sullivan DD, Seltzer J, Rossing TH, Drazen JM, Reid LM 1985 Density gradient study of bronchial mucus aspirates from healthy volunteers (smokers and non-smokers) and from patients with tracheostomy. Exp Lung Res 9:289-308

9. Bhaskar KR, O'Sullivan DD, Hincman HO, Reid LM, Coles SJ 1986 Density gradient analysis of secretions produced in vitro by human and canine airway mucosa: identification of lipids and proteoglycans in such secretions. Exp Lung Res 10:401422

10. Boat TF, Cheng PW, Iyer RN, Carlson DM, Polony I 1976 Human respiratory tract secretions: mucous glycoproteins of nonpurulent tracheobronchial secretions, and sputum of patients with bronchitis and cystic fibrosis. Arch Biochem Biophys 177:95-104

11. Boat TF, Cheng PW, Wood RE 1976 Tracheobronchial mucus secretion in vivo and in vitro by epithelial tissues from cystic fibrosis and control subjects. Mod Probl Paediatr 19:141-152

12. LeTreut A, Lamblin G, Leray L, Guenet L, Filliat M, Roussel P 1984 Identification of degraded proteoglycans in the sputum of patients suffering from chronic bronchial hypersecretion. In: Lawson D (ed) Cystic Fibrosis: Horizons. John Wiley and Sons, NewYork, p 347

13. Gallagher JT, Kent PW 1975 Structure and metabolism of glycoproteins and glycosaminoglycans secreted by organ cultures of rabbit trachea. Biochem J 148:187-196

14. Paul A, Picard J, Mergey M, Veissiere D, Finkbeiner WE, Basbaum CB 1988 Glycoconjugates secreted by bovine tracheal serous cells in culture. Arch Biochem Biophys 260:75-84

15. Cheng PW, Boat TF, Cranfill JR, Yankaskas RC 1989 Increased sulfation of glycoconjugates by cultured nasal epithelial cells from patients with cystic fibrosis. J Clin Invest 84:68-72

16. Basbaum CB, Forsberg LS, Paul A, Sommerhoff C, Finkbeiner WE 1987 Studies of tracheal secretion using serous cell cultures and monoclonal antibodies. Biorheology 24:585-588 
17. Rahmoune H, Lamblin G, Lafitte JJ, Galabert C, Filliat M, Roussel P 1991 Chondroitin sulfate in sputum from patients with cystic fibrosis and chronic bronchitis. Am J Respir Cell Mol Biol 5:315-320

18. Frates RC, Kaizu T, Last JA 1983 Mucous glycoproteins secreted by respiratory epithelial tissue from cystic fibrosis patients. Pediatr Res 17:30-34

19. Mohapatra NK, Cheng PW, Parker JC, Paradiso AM, Yankaskas JR, Boucher RC, Boat TF 1995 Alteration of sulfation of glycoconjugates, but not sulfate transport and intracellular inorganic sulfate content in cystic fibrosis airway epithelial cells. Pediatr Res 38:42-48

20. Bhaskar KR, Turner BS, Grubman SA, Jefferson DM, LaMont JT 1998 Dysregulation of proteoglycan production by intrahepatic biliary epithelial cells bearing defective $(\Delta \mathrm{F} 508)$ cystic fibrosis transmembrane conductance regulator. Hepatology 27:7-14

21. Kjellen L, Lindahl U 1991 Proteoglycans: structures and interactions. Annu Rey Biochem 60:443-475

22. Stockley RA, Bayley D, Hill SL, Hill AT, Crooks S, Campbell EJ 2001 Assessment of airway neutrophils by sputum colour: correlation with airways inflammation. Thorax 56:366-372

23. Bertolotto A, Palmucci L, Gagliano A, Mongini T, Tarone G 1986 Immunohistochemical localization of chondroitin sulfate in normal and pathological human muscle. J Neurol Sci 73:233-244

24. Koshino T, Bhaskar KR, Reid LM, Gerard C, Warner A, Shore SA, Anderson K, Butler G, IIjima H, Drazen JM 1990 Recovery of an epitope recognized by a novel monoclonal antibody from airway lavage during experimental induction of chronic bronchitis. Am J Respir Cell Mol Biol 2:453-462

25. Carlstedt I, Lindgren H, Sheehan JK 1983 The macromolecular structure of human cervical-mucus glycoproteins. Studies on fragments obtained after reduction of disulphide bridges and after subsequent trypsin digestion. Biochem J 213:427-435

26. Sajjan U, Reisman J, Doig P, Irvin RT, Forstner G, Forstner J 1992 Binding of non-mucoid Pseudomonas aeruginosa to normal human intestinal mucin and respiratory mucin from patients with cystic fibrosis. J Clin Invest 89:657-665

27. Redinbaugh MG, Turley RB 1986 Adaptation of the bicinchoninic acid protein assay for use with microtiter plates and sucrose gradient fractions. Anal Biochem 53:267271

28. Cappelletti R, Rosso MD, Chiarugi VP 1979 A new electrophoretic method for the complete separation of all known glycosaminoglycans in a monodimensional run. Anal Biochem 99:311-315

29. Engelhardt JF, Yankaskas JR, Ernst SA, Yang Y, Marino CR, Boucher RC, Cohn JA Wilson JM 1992 Submucosal glands are the predominant site of CFTR expression in the human bronchus. Nat Genet 2:240-248

30. Brahimi-Horn MC, Deudon E, Paul A, Mergey M, Mailleau C, Basbaum C, Dohrman A, Capeau J 1994 Identification of decorin proteoglycan in bovine tracheal serous cells in culture and localization of decorin mRNA in situ. Eur J Cell Biol 64:271-280

31. Shirk RA, Parthasarathy N, San Antonio JD, Church FC, Wagner WD 2000 Altered dermatan sulfate structure and reduced heparin cofactor II-stimulating activity of biglycan and decorin from human athersclerotic plaque. J Biol Chem 275:1808518092

32. Goldstein, W Doring G 1986 Lysosomal enzymes from polymorphonuclear leucocytes and proteinase inhibitors in patients with cystic fibrosis. Am Rev Respir Dis 134:49-56

33. Suter S, Schaad UB, Tegner H, Ohlsson K, Desgrandchamps D, Waldvogel FA 1986 Levels of free granulocyte elastase in bronchial secretions from patients with cystic fibrosis: effects of antimicrobial treatment against Pseudomonas aeruginosa. J Infect Dis 153:902-909

34. Houdret N, Ramphal R, Scharfman A, Perini J, Filliat M, Lamblin G, Roussel P 1989 Evidence for the in vivo degradation of human respiratory mucins during Pseudomonas aeruginosa infection. Biochim Biophys Acta 992:96-105

35. Hascall VC, Riolo RL, Howard JJ, Reynolds CC 1972 Treatment of bovine nasa cartilage proteoglycan with chondroitinase from Flavobacterium heparinum and Proteus vulgaris. J Biol Chem 247:4521-4528

36. Bhaskar KR, Brown R, O'Sullivan DD, Melia S, Duggan M, Reid L 1991 Bronchial mucus hypersecretion in acute quadriplegia: macromolecular yields and glycoconjugate composition. Am Rev Resp Dis 143:640-648

37. Forstner JF, Oliver MG, Sylvester FA 1995 Production, structure, and biologic relevance of gastrointestinal mucins. In: Blaser MJ, Smith PD, Ravdin JI, Greenberg HB, Guerrant HB (eds) Infections of the Gastrointestinal Tract. Raven Press, New York, pp 71-88

38. Yang H, Farooqui AA, Horrocks LA 1994 Effects of glycosaminoglycans and glycosphingolipids on cytosolic phospholipases A2 from bovine brain. Biochem J 299:91-95

39. Ruoslahti E 1989 Proteoglycans in cell regulation. J Biol Chem 264:13369-13372

40. Basbaum CB, Finkbeiner WE 1988 Airway secretion: a cell specific analysis. Horm Metab Res 20:661-667

41. Parmley RT, Rahemtulla F, Cooper MD, Roden L 1985 Ultrastructural and biochemical characterization of glycosaminoglycans in HNK-1 positive large granular lymphocytes. Blood 66:20-25

42. Rothenberg ME, Pomerantz JL, Owen Jr WF, Avraham S, Soberman RJ, Austen KF, Stevens RL 1988 Characterization of a human eosinophil proteoglycan, and augmentation of its biosynthesis and size by interleukin 3, interleukin 5 and granulocyte/ macrophage colony stimulating factor. J Biol Chem 263:13901-13908

43. Hunningshake GW, Gadek JE, Kavanami O, Ferrans VJ, Crystal RG 1979 Inflammatory and immune processes in the human lung in health and disease: evaluation by bronchoalveolar lavage. Am J Pathol 97:149-206

44. Sommerhoff CP, Nadel JA, Basbaum CB, Caughey GH 1990 Neutrophil elastase and cathepsin $\mathrm{G}$ stimulate secretion from cultured bovine airway gland serous cells. J Clin Invest 85:682-689

45. Roussel P, Lamblin G, Degand P 1975 Heterogeneity of the carbohydrate chains of sulfated bronchial glycoproteins isolated from a patient suffering from cystic fibrosis. J Biol Chem 250:2114-2122

46. Chace KV, Leahy DS, Martin R, Carubelli R, Flux M, Sachdev GP 1983 Respiratory mucous secretions in patients with cystic fibrosis: relationship between levels of highly sulfated mucin component and severity of the disease. Clin Chim Acta 132:143-155

47. Dasgupta B, King M 1996 Reduction in viscoelasticity of cystic fibrosis sputum in vitro with combined treatment by nacystelyn and rhDNase. Pediatr Pulmonol 22:161166

48. Vasconcellos CA, Allen PG, Wohl M, Drazen JM, Janmey PA 1994 Reduction in sputum viscosity of cystic fibrosis sputum in vitro by gelsolin. Science 263:969-971

49. Feng W, Garrett H, Speert DP, King M 1998 Improved clearability of cystic fibrosis sputum with dextran treatment in vitro. Am J Respir Crit Care Med 157:710-714 